\title{
Elemental Identification by Combining Atomic Force Microscopy and Kelvin Probe Force Microscopy
}

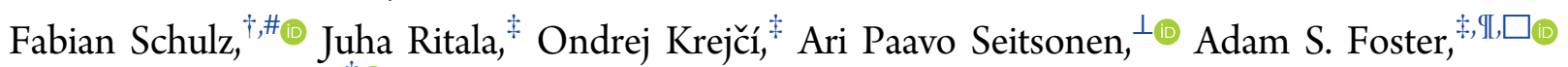
and Peter Liljeroth $*+\dagger$

${ }^{\dagger}$ Department of Applied Physics, Aalto University School of Science, P.O. Box 15100, FI-00076 Aalto, Finland

${ }^{\ddagger}$ COMP Center of Excellence, Department of Applied Physics, Aalto University School of Science, P.O. Box 11100, FI-00076 Aalto, Finland

${ }^{\perp}$ Département de Chimie, École Normale Supérieure, 24 rue Lhomond, F-75005 Paris, France

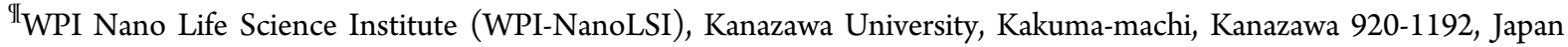

$\square$ Graduate School Materials Science in Mainz, Staudinger Weg 9, D-55128 Mainz, Germany

Supporting Information

ABSTRACT: There are currently no experimental techniques that combine atomic-resolution imaging with elemental sensitivity and chemical fingerprinting on single molecules. The advent of using molecular-modified tips in noncontact atomic force microscopy (nc-AFM) has made it possible to image (planar) molecules with atomic resolution. However, the mechanisms responsible for elemental contrast with passivated tips are not fully understood. Here, we investigate elemental contrast by carrying out both nc-AFM and Kelvin probe force microscopy (KPFM) experiments on epitaxial monolayer hexagonal boron nitride $(\mathrm{hBN})$ on $\operatorname{Ir}(111)$. The $\mathrm{hBN}$ overlayer is inert, and the in-plane bonds connecting nearest-neighbor boron and nitrogen atoms possess strong covalent character and a bond length of only $\sim 1.45 \AA$. Nevertheless, constant-height maps of both the frequency shift $\Delta f$ and the local contact potential difference exhibit striking sublattice asymmetry. We match the different atomic sites with the observed contrast by comparison with nc-AFM image simulations based on the density functional theory optimized hBN/ $\operatorname{Ir}(111)$ geometry, which yields detailed information on the origin of the atomic-scale contrast.

KEYWORDS: hexagonal boron nitride, noncontact atomic force microscopy (nc-AFM), Kelvin probe force microscopy (KPFM), elemental contrast, van der Waals density functional theory

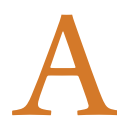

tomic-resolution microscopies are key enabling techniques in modern materials research. These techniques include scanning probe microscopies (scanning tunneling microscopy, STM, and noncontact atomic force microscopy, nc-AFM) $)^{1-3}$ as well as high-resolution transmission electron microscopy (e.g., scanning transmission electron microscopy, STEM). ${ }^{4}$ Identifying different elements is more challenging, especially based purely on experimental results. ${ }^{5-9}$ Modern, aberration-corrected electron microscopes make it possible to chemically fingerprint different elements through atomic-resolution electron energy loss spectroscopy $(\mathrm{EELS})^{8-11}$ or $Z$-contrast in the annular dark field imaging mode. $^{12}$ These techniques, when coupled with $a b$ initio calculations, can also yield information on chemical bonding configuration down to the single-atom level. ${ }^{10,11}$ However, electron microscopy has demonstrated chemical sensitivity only on solid state materials. Due to the high-energy electron beam, STEM has not yet reached atomic structural or chemical resolution on more sensitive structures such as small organic molecules. On the other hand, scanning probe microscopy can be used to image small molecules with atomic resolution. ${ }^{13,14}$

When operated in the frequency modulation mode, ${ }^{15} \mathrm{nc}$ AFM measures atomic-scale forces between the tip on an oscillating cantilever and the sample surface through changes of the resonance frequency $(\Delta f)$ of the cantilever. nc-AFM can yield atomic resolution, which has been amply demonstrated on elemental semiconductors as well as on heteroatomic surfaces of compound semiconductors and polar insulators such as alkali halides and oxides. ${ }^{1,3,6,16-18}$ On such heteroatomic surfaces, typically only one type of atom is actually imaged with a given tip, because the polar nature of the compounds results in a strong variation in the short-range forces above the negatively

Received: December 20, 2017

Accepted: May 25, 2018

Published: May 25, 2018 


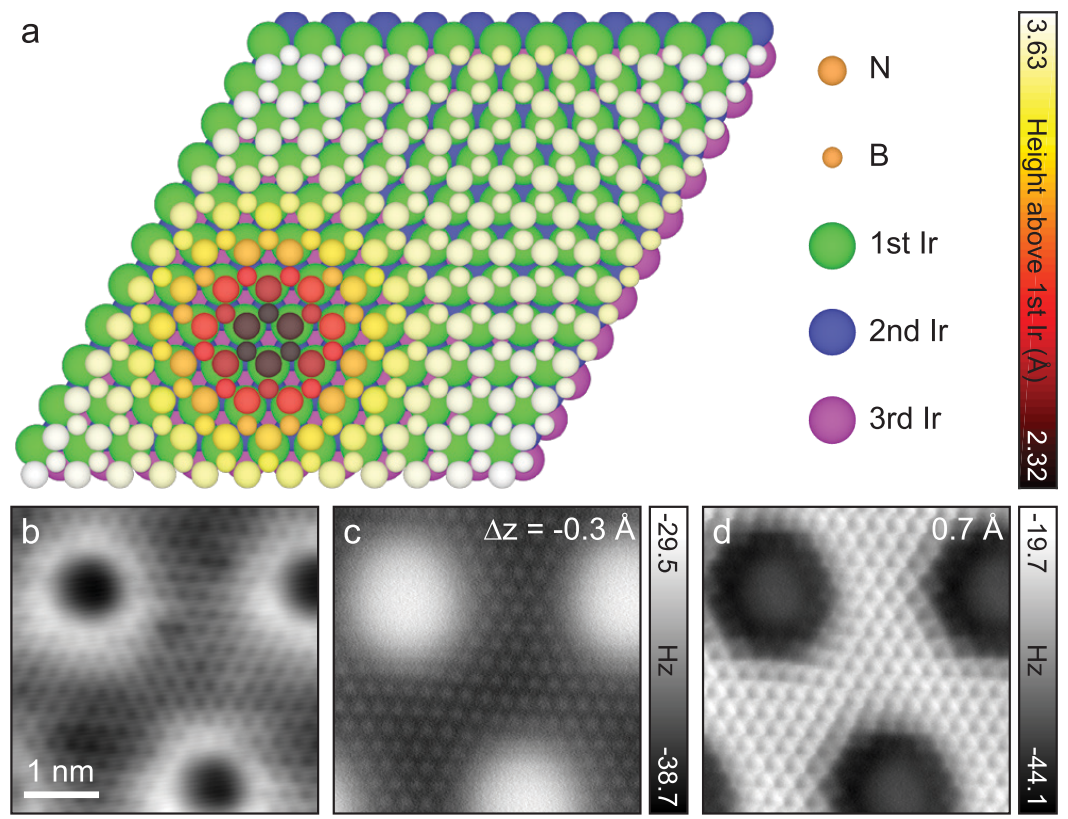

Figure 1. (a) DFT-optimized structure of hBN/Ir(111). (b) Constant-current STM image of hBN/Ir(111). Set point: $0.10 \mathrm{~V}, 0.31 \mathrm{nA}$. (c and d) Constant-height nc-AFM images of $\mathrm{hBN} / \mathrm{Ir}(111)$ with CO-passivated tip at (c) large and (d) small tip-sample distance. $\Delta z$ as defined in the main text. Set point: $0.10 \mathrm{~V}, 0.31 \mathrm{nA}$.

and positively charged atoms. ${ }^{5,19}$ Additional information can be gained from force spectroscopy, i.e., the measurement of $\Delta f$ as a function of tip-sample distance $z$. In a seminal contribution, Sugimoto et al. identified different atomic species in a disordered surface alloy on $\mathrm{Si}(111)$ by comparing the maximum attractive forces on different lattice sites. ${ }^{7}$ This methodology has recently been extended to estimating electronegativities of surface atoms. ${ }^{20}$ In addition to the force channel, another avenue for achieving elemental contrast is to measure the local contact potential difference (LCPD) by Kelvin probe force microscopy (KPFM). ${ }^{21-27}$ Nevertheless, contrast between different elements on the surface has thus far only been observed for highly polar or reactive surfaces.

Atomic-resolution nc-AFM studies can be extended to molecular systems through chemical passivation of the tip apex, e.g., by controlled pick-up of a single carbon monoxide (CO) molecule. ${ }^{13,14,28-36}$ With these tips, it is possible to enter a regime where the tip-sample interaction is dominated by the Pauli repulsion between the last atom of the tip and the sample atom directly under it. ${ }^{28,37-39}$ In addition to molecules, this technique has been used to measure atomic positions and surface corrugations of two-dimensional materials (e.g., graphene and hexagonal boron nitride)..$^{32,40-44}$

Achieving chemical sensitivity with passivated tips is a more delicate issue, as bond formation with the sample similar to reactive tips is suppressed. Still, changes in the total electron density and electrostatic forces on different atoms are expected to contribute to the image contrast. ${ }^{18,37,45-48}$ Consequently, passivated tips enabled elemental contrast also on molecular systems, in both $\Delta f^{29,49-52}$ and LCPD. ${ }^{50,53}$ However, cross-talk between perceived elemental contrast and nonplanar topography, edge effects, and resulting image distortions due to flexibility of the tip apex make systematic studies in molecular systems challenging. ${ }^{34,35,51,52,54-56}$ At present, chemical fingerprinting by scanning probe microscopy remains an elusive goal.

Here, we demonstrate elemental contrast in both $\Delta f$ and LCPD on a covalently bonded system, monolayer hexagonal boron nitride (hBN). This system is well-defined on the atomic level and does not suffer from topographic corrugation or edge effects. We employ nc-AFM with CO-functionalized tips ${ }^{28}$ to investigate the atomic-scale contrast on epitaxial hBN on $\operatorname{Ir}(111){ }^{57}$ Despite the mostly covalent character of the B-N bond $^{58,59}$ and a nearest-neighbor distance of only $\sim 1.45 \AA$, constant-height maps of both $\Delta f$ and LCPD acquired over $\mathrm{hBN} / \operatorname{Ir}(111)$ exhibit striking sublattice asymmetry. nc-AFM image simulations based on the density functional theory (DFT)-optimized $\mathrm{hBN} / \operatorname{Ir}(111)$ geometry allow us to match the two distinct atomic sites with the boron and the nitrogen sublattices. Studies on such clean model systems are essential to shine light on the origin of atomic-scale contrast in nc-AFM on surfaces and molecular systems.

\section{RESULTS AND DISCUSSION}

Figure 1a shows the DFT-optimized structure of our model surface, monolayer hBN on $\operatorname{Ir}(111)$ (see Methods for computational details). The lattice mismatch between $\mathrm{hBN}$ and the iridium surface results in a periodic variation of the stacking between boron and nitrogen and substrate atoms, thus creating a moiré superstructure with a periodicity of approximately $30 \AA$. The different registries dictate the interaction strength between the $\mathrm{hBN}$ layer and the metallic surface, giving rise to a structural corrugation of the overlayer of $\sim 1.3 \AA$, as well as a modulation of the local work function within the moire unit cell. ${ }^{57}$ However, the regions surrounding the moire depressions are essentially flat.

Figure $1 \mathrm{~b}$ is an atomically resolved STM image of hBN grown on $\operatorname{Ir}(111)$ by chemical vapor deposition (see Methods for experimental details), acquired with a CO-passivated tip prepared on a $\mathrm{Cu}(111)$ surface $(\mathrm{CO} / \mathrm{Cu}$ tip) (all data shown here were acquired with $\mathrm{CO} / \mathrm{Cu}$ tips). The topography highlights the depressions of the superstructure and alignment of the hBN lattice with the moire unit cell, in good agreement with the DFT calculations and previous studies. ${ }^{5,60}$ Two constant-height nc-AFM images, recorded at different tip- 

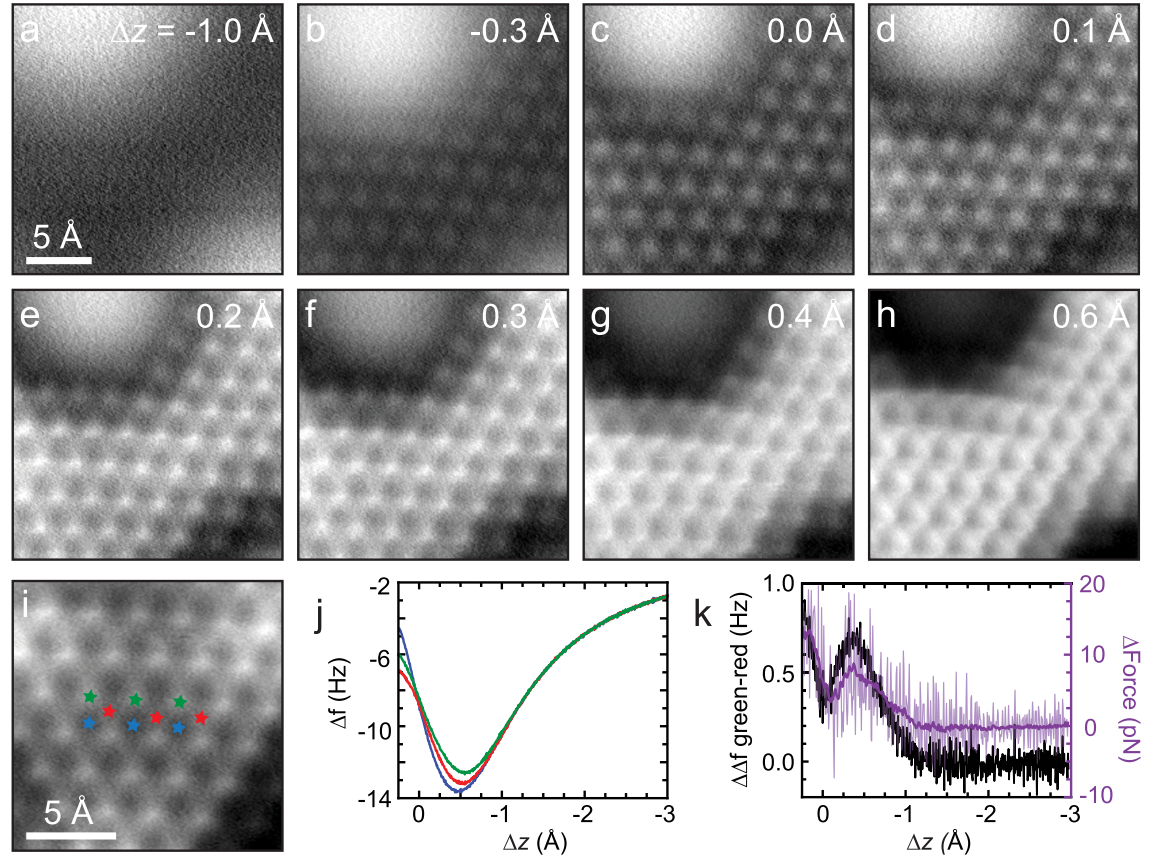

Figure 2. $(\mathrm{a}-\mathrm{h})$ Distance-dependent constant-height nc-AFM images with decreasing tip-sample distance. Set point: $0.10 \mathrm{~V}, 0.31 \mathrm{nA}$. (i) Constant-height nc-AFM image indicating the positions of $\Delta f(z)$ spectra. (j) Average $\Delta f(z)$ spectra for the two inequivalent top sites (green, red) and the hollow site (blue), calculated from individual spectra taken at the positions marked in panel (i). Set point: $0.05 \mathrm{~V}, 0.30 \mathrm{nA}$. (k) $\Delta f(z)$ difference between the two top sites (green - red = black) and the corresponding force difference (magenta, semitransparent: raw data; solid: adjacent-averaged). Note that data in $(\mathrm{a})-(\mathrm{h})$ are taken with a different $\mathrm{CO} / \mathrm{Cu}$ tip than data in (i) $-(\mathrm{k})$.

sample distances $\Delta z$ and with the same CO-passivated tip, are shown in Figures 1c,d. Throughout the article, $\Delta z$ refers to the relative tip-sample approach with respect to the STM feedback set point. Thus, negative $\Delta z$ increases the tip-sample distance and positive $\Delta z$ decreases it. In both images, two different levels of contrast can be distinguished, corresponding to the moiré and hBN lattices. At large distances (panel c, $\Delta z=-0.3 \AA$ ), the depressions of the moiré appear with a less negative $\Delta f$. Similarly, the atomic contrast comprises a hexagonal lattice of top sites with less negative $\Delta f$. Both contrasts seem to reverse at small tip-sample distances (panel d, $\Delta z=0.7 \AA$ ), where the moire depressions exhibit more negative $\Delta f$, and at the atomic scale, a honeycomb lattice of less negative $\Delta f$ appears.

In the limit of small amplitudes, for which the frequency shift is given by $\Delta f=-f_{0} / 2 k . \partial_{z} F_{z}$ ( $F_{z}$ is the vertical component of the tip-sample force ${ }^{1}$ and $f_{0}$ and $k$ are the resonance frequency and stiffness of the cantilever, respectively), more negative $\Delta f$ values can be interpreted as more attractive tip-sample forces. Then, the moire contrast reversal is easily explained: At large tip-sample distance, the depressions are less attractive than the surrounding regions, while at close distances they are less repulsive [see Supporting Information (SI) for comparison of the frequency shift as a function of tip-sample distance between the moiré depression and the surrounding region].

Understanding the atomic-scale features requires a more careful inspection, as a mere reversal from attractive to repulsive contrast is not expected for CO-passivated tips. ${ }^{32}$ Figure $2 \mathrm{a}-\mathrm{h}$ show a series of distance-dependent constant-height nc-AFM images, covering a $z$-range of $1.6 \AA$. At very large distances (panel a), no atomic contrast is observed, but only the longrange moiré corrugation. Approaching the surface (panel b), the hexagonal pattern of less negative $\Delta f$ appears, similarly to Figure 1c. On further reducing the tip-sample distance (panels c-e), every second hollow site starts to exhibit less negative $\Delta f$ as well, such that a lattice with three distinct sites forms. At very close distances (panels $\mathrm{f}-\mathrm{h}$ ), the intensities of the two sites with the less negative $\Delta f$ approach each other and equalize. Because the two sites have different sizes, this gives rise to a hexagonal lattice of repulsive triangles, which we interpret as the atomic lattice of the hBN. Thus, the sites of less negative $\Delta f$ observed at large distances (Figure $2 b$ ) do not correspond to the hollow sites of the atomic hBN lattice appearing less attractive but one of the two top sites appearing more repulsive. We have reproduced this evolution of the atomic contrast with several macroscopically different $\mathrm{CO}$ tips prepared on $\mathrm{Cu}(111)$, as well as with CO tips prepared on $\operatorname{Ir}(111)$ [see SI for images acquired with a CO tip prepared on $\operatorname{Ir}(111)]$. The observed contrast is thus intrinsic and not related to tip artifacts.

The above observations reveal a clear asymmetry between the boron and nitrogen sublattices in nc-AFM images of $\mathrm{hBN}$. We can quantify this asymmetry by measuring the frequency shift as a function of tip-sample distance $[\Delta f(z)]$, as depicted in Figures 2i,j. The graph in Figure $2 \mathrm{j}$ shows $\Delta f(z)$ spectra for the three distinct sites, each of which is the average of three equivalent locations as marked in Figure 2i (see SI for the individual spectra). In Figure $2 \mathrm{k}$, we plot the difference (black) between the two inequivalent top sites (green, red) of the hBN lattice and the corresponding force difference (magenta) recovered from $\Delta f$ via the Sader-Jarvis method. ${ }^{61}$ Taking the adjacent-averaged force data, the difference between the boron and nitrogen sublattice at typical imaging distances is no more than $10 \mathrm{pN}$. Note that the $\Delta f(z)$ approach curves go to smaller tip-sample distances than the images in Figure $2 \mathrm{a}-\mathrm{h}$, but we observed instabilities of the tip-sample junction when imaging at such small distances.

In order to match the two sublattices with the boron and nitrogen atoms, we simulate nc-AFM images using the MechAFM code, ${ }^{62}$ which is based on a molecular mechanics 

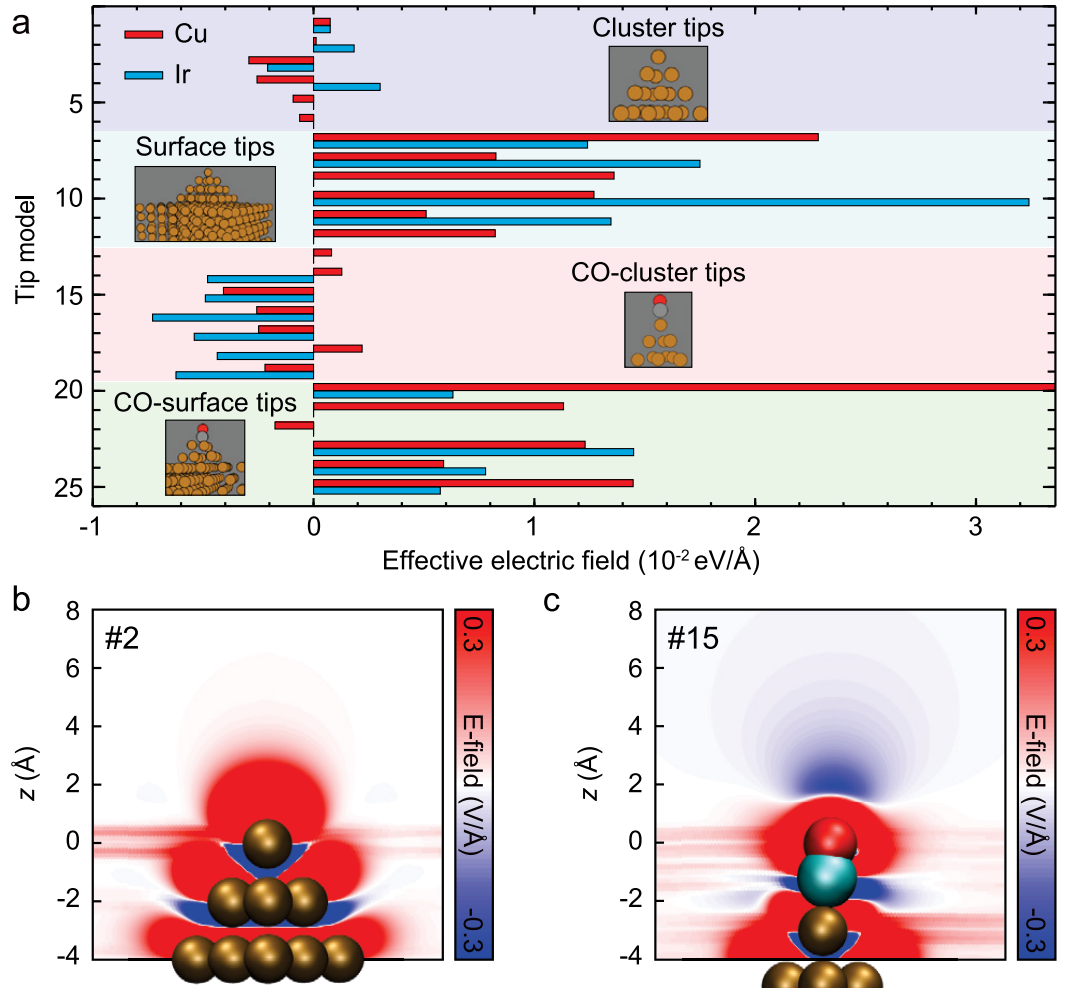

C
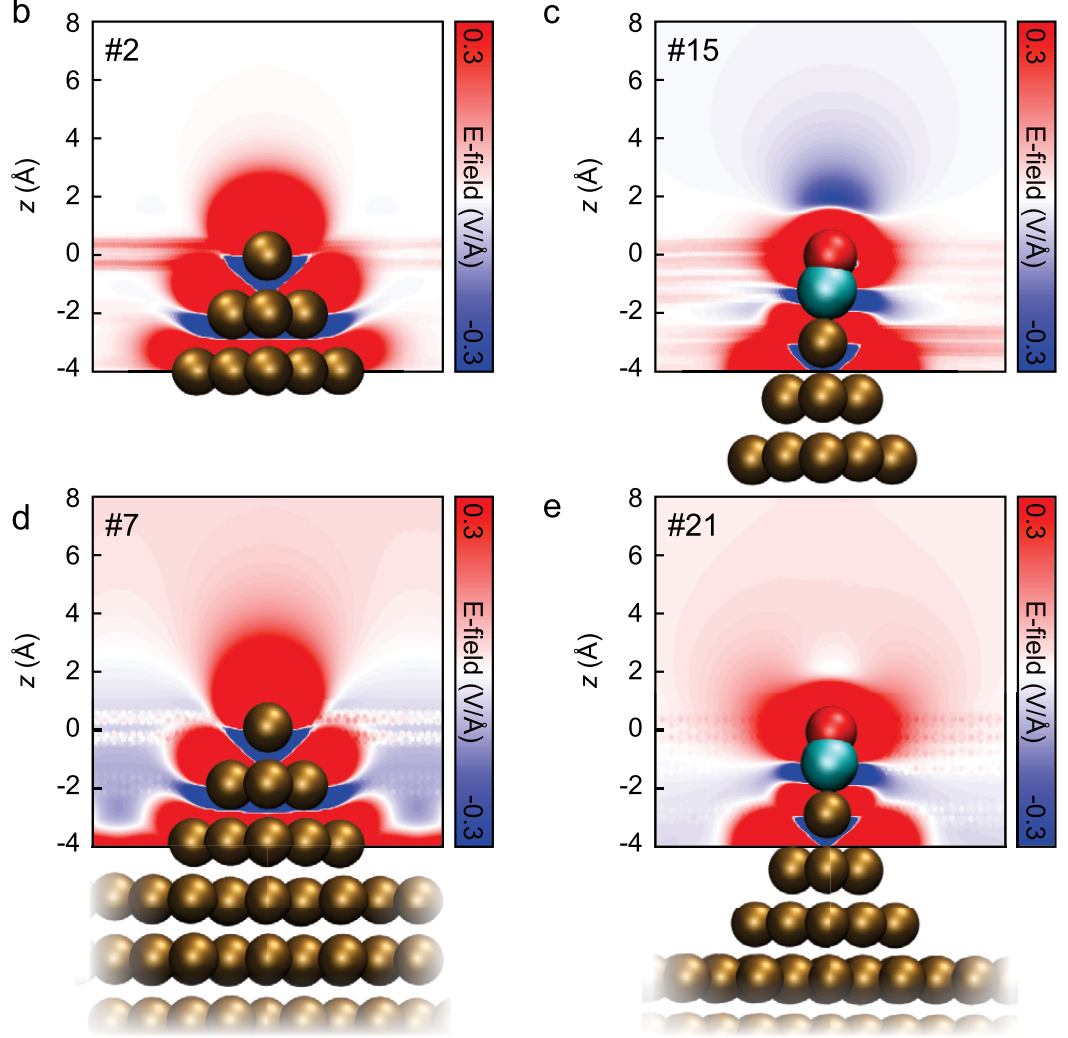

Figure 3. (a) Effective electric field for various tip models extracted from DFT. (b-e) Example structure and its electric field for a (b) cluster tip, (c) CO-cluster tip, (d) surface tip, and (e) CO-surface tip.

model taking into account the flexibility of the $\mathrm{CO}$ molecule at the tip apex ${ }^{34,35,41}$ and the atomic coordinates from the DFToptimized hBN/Ir(111) structure (see Methods for details of the nc-AFM simulations). Recent studies have shown the importance of electrostatic forces in understanding AFM image contrast, ${ }^{46,47}$ and thus they need to be taken into account as well. However, there are contradicting reports regarding the charge associated with CO-passivated metal tips. ${ }^{18,35,45,46,48}$ One issue is that $a b$ initio simulations of such tips usually focus on unrealistically small tip models, often containing none ${ }^{28,37,39}$ or only a few metal atoms $s^{30,31,38,48}$ in addition to the $\mathrm{CO}$ molecule. We address this problem by employing DFT to simulate more realistic tip models, which also contain a metallic surface to account for the bulk tip ${ }^{56}$ (see Methods for computational details).

In Figure 3a, we plot the effective long-range electrostatic field of the tip extracted from our DFT calculations for several
$\mathrm{CO} / \mathrm{Cu}$ and $\mathrm{CO} / \mathrm{Ir}$ tip models. The different models are grouped into four classes: (i) purely metal, pyramidal clusters ("cluster tips"), (ii) metal clusters on a metal surface ("surface tips"), (iii) $\mathrm{CO}$ on a metal cluster ("CO-cluster tips"), and (iv) $\mathrm{CO}$ on a metal cluster on a metal surface ("CO-surface tips"). An example tip and its actual electric field is shown for each of the four classes in Figure $3 b-e$ (see SI for the structures of all calculated tips). In order to clearly demonstrate the effect of the $\mathrm{CO}$ molecule and $\mathrm{Cu}$ surface on the electric field, all example tips have the same 10-atom $\mathrm{Cu}$ cluster. The cluster tip in Figure $3 \mathrm{~b}$ exhibits a positive electric field, as is commonly assumed for metallic tips. ${ }^{18,45,48}$ However, Figure $3 \mathrm{a}$ indicates that within DFT, not just the magnitude but also the sign of this field depend on the precise cluster geometry. For surface tips, we find consistently a positive electric field at the apex, as demonstrated in Figure 3d. Adding a $\mathrm{CO}$ to a $\mathrm{Cu}$ cluster tip, as shown in Figure $3 c$, results in a negative electric field at the 

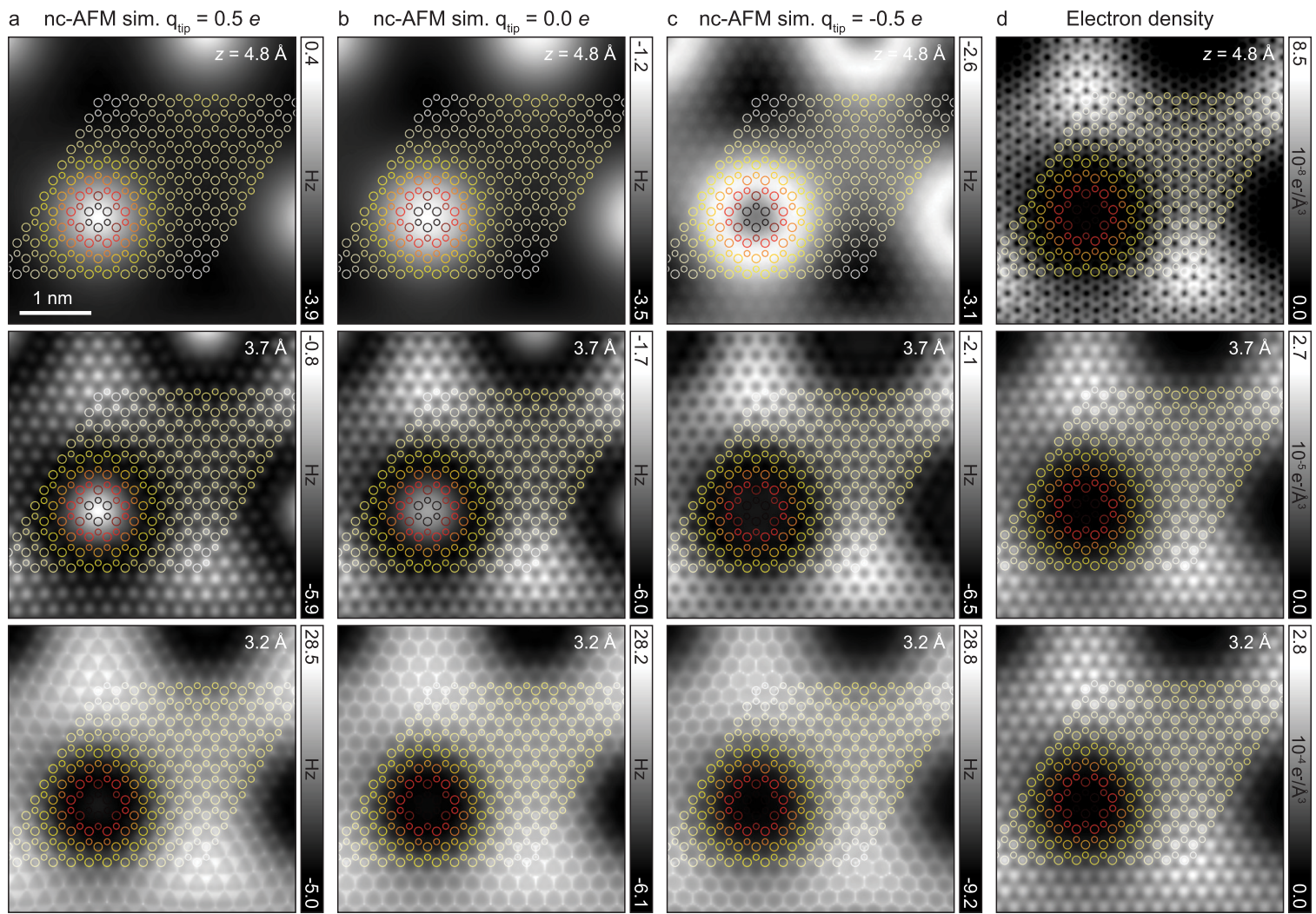

Figure 4. (a-c) nc-AFM image simulations for (a) positively charged, (b) neutral, and (c) negatively charged CO tip at different tip-sample distances, with overlaid hBN lattice. Large circles: nitrogen, small circles: boron. The $z$ values indicate the height of the unrelaxed oxygen atom with respect to the mean height of the hBN layer. (d) Constant-height electron density as calculated by DFT.

apex, in agreement with previous DFT calculations for a COcluster tip. $^{48}$ Indeed, we find that most of the CO-cluster tips exhibit a negative effective electric field. In contrast, Figure 3a reveals that within the most realistic subset of CO-surface tips, all but one exhibit a positive effective electric field. This positive electric field is due to the bulk metallic tip and results from the Smoluchowski effect, and this is the dominant long-range contribution. We observe a negative field at around $2 \AA$ from the oxygen apex (see Figure $3 \mathrm{e}){ }^{48}$ but the extent of this negative component is exaggerated for cluster tips as seen in Figure $3 c$, due to the multiple exposed apexes and their unphysical impact on the Smoluchowski effect. In the more realistic CO-surface tip models used here, the field rapidly becomes positive, as shown in Figure 3e. The electric field for the CO-surface tip shown in Figure 3e agrees qualitatively well with the semiempirical $\mathrm{CO}$ tip model introduced by Ellner et al., ${ }^{48}$ which was fitted to reproduce the nc-AFM contrast of a $\mathrm{Cl}$ vacancy in $\mathrm{NaCl}$. However, in their model the negative electric field close to the oxygen extends more into the vacuum compared to our CO-surface tips before it becomes positive. Our finding applies to both $\mathrm{Cu}$ and $\mathrm{Ir}$ tips, suggesting that this is a general feature of $\mathrm{CO} /$ metal tips. Calculation of the dipole associated with the whole tip instead of the effective electric field yields qualitatively identical results, and the conclusions do not depend on which method is used (see SI for a plot of the dipole for all simulated tip models). Hence, the simplest reasonable approximation is to consider the $\mathrm{CO}$ tip as carrying a positive partial charge. This charge then interacts with the electrostatic potential of the $\mathrm{hBN} / \operatorname{Ir}(111)$ sample obtained from DFT, thus accounting for the electrostatic forces in our nc-AFM simulations. Despite its simplicity, such an approach has previously shown to yield excellent agreement with experimental nc-AFM images. ${ }^{14,47}$

Three large-scale, simulated nc-AFM images of hBN/Ir(111) at different tip-sample distances and for a positively charged CO tip $\left(q_{\text {tip }}=0.5 e\right)$ are shown in Figure 4a. Surprisingly, the image simulations suggest that the boron sublattice appears first in the constant-height images, with the nitrogens becoming visible only as the tip further approaches the $\mathrm{hBN}$ layer.

For comparison, we also simulated nc-AFM images with a neutral $\left(q_{\text {tip }}=0.0 e\right)$ and negatively charged $\left(q_{\text {tip }}=-0.5 e\right)$ tip, as shown in Figure $4 \mathrm{~b}$ and c, respectively. The main observations are as follows: Even when neglecting electrostatic interactions as for the neutral tip, there is a slight asymmetry between the boron and nitrogen sublattice, with the former appearing first at large tip-sample distances. This atomic contrast is thus due to short-range interactions, which are described by Lennard-Jonestype pair potentials in the nc-AFM simulation, and is in agreement with the larger atomic radius of boron compared with nitrogen, ${ }^{63}$ which causes an earlier onset of repulsive forces. This asymmetry then becomes more pronounced with a positively charged tip, which also causes strong distortions of the atomic honeycomb lattice at small tip-sample distances, such that it appears as a hexagonal lattice of repulsive triangles. This contrast is found neither for the neutral nor for the negatively charged tip. On the contrary, the negatively charged tip yields a nearly perfect honeycomb lattice at such small distances. At large tip-sample distances, it shows the nitrogens first and, importantly, also causes a reversal of the moire contrast, which is not observed for the other two tips. Thus, by far the best agreement with the experimental data is achieved 
for the positively charged tip (see SI for image simulations at additional heights).

The fact that the boron sublattice is observed first is rather counterintuitive: As repulsive atomic contrast is dominated by the Pauli repulsion, one would expect the element exhibiting the higher charge density to appear first upon approaching the surface. Figure $4 \mathrm{~d}$ shows constant-height slices of the electron density from our DFT calculations, at the heights of the tip's oxygen atom in the image simulations. While the slice at largest distance has significant contributions of the underlying Ir substrate (note also the very small scale of the changes in the electron density), at the two smaller tip-sample distances, the nitrogens clearly exhibit a higher electron density. Thus, our ncAFM images contradict the conventional interpretation, which highlights the limitations of relying only on the $\Delta f$ channel. $^{24}$

Figure $5 \mathrm{a}-\mathrm{h}$ display a full series of simulated nc-AFM images for the positively charged $\mathrm{CO}$ tip, covering a total range of tip-
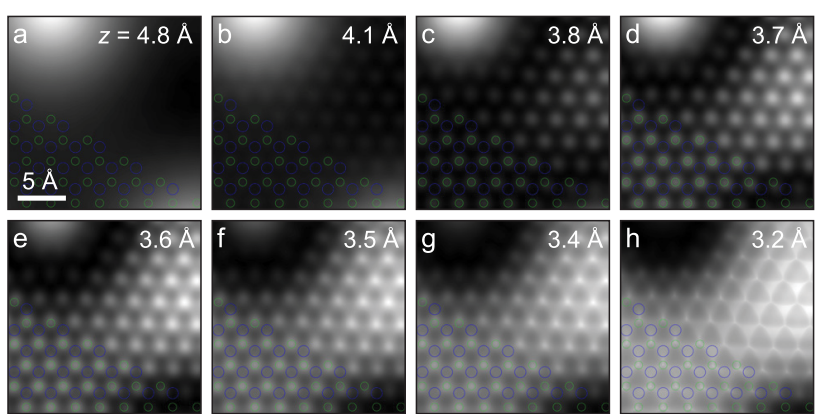

Figure 5. (a-h) nc-AFM image simulations for a positively charged CO tip for different tip-sample distances, with overlaid hBN lattice. Large blue circles: nitrogen; small green circles: boron. The $z$ values indicate the height of the unrelaxed oxygen atom with respect to the mean height of the $\mathrm{hBN}$ layer.

sample distances of $1.6 \AA$, the same as in the experimental series (Figure 2a-h). The detailed evolution of the moire and atomic contrast is in excellent agreement: The theoretical images reproduce all three observed atomic contrasts, as well as the relative range of tip-sample distances at which they appear. In addition, the simulation indicates that only at closest tipsample distances (Figure 3i) is the contrast formation strongly influenced by the bending of the $\mathrm{CO}$ at the tip apex, while this effect is negligible for images taken farther away. It is noteworthy that even though it is well-established that bulk metallic tips carry a positive electric field, ${ }^{48,64}$ our finding that it also influences the atomic contrast with CO-terminated tips is surprising with respect to previous studies. ${ }^{48}$ In fact, based on the study of the nc-AFM contrast of $\mathrm{NaCl}$ and a $\mathrm{Cl}$ vacancy, it was shown that the more complicated multipole character of the CO-tip electric field can be relevant in more strongly ionic systems $^{48}$ (see SI for details). We have tested this in the case of $\mathrm{hBN}$ by carrying out additional image simulations. We used a modified version of the probe-particle model, which calculates the electrostatic forces based on a tip that consists of a positive dipole accounting for the bulk metallic tip and a small negative quadrupole moment for the CO (see SI for details of the ncAFM simulations). These simulations qualitatively agree with our simpler model and also suggest that the boron lattice is imaged first at larger tip-sample distances (see SI for the simulated images and additional discussion). The fact that already the simple approximation of a positive point charge yields excellent agreement with experiments in the case of hBN is most likely due to the different bonding character compared to $\mathrm{NaCl}$. In the former, the bonds have only small polar character, ${ }^{59}$ and thus the electric field associated with the partial atomic charges extends less into the vacuum (in the case of monolayer $\mathrm{hBN} / \operatorname{Ir}(111)$, the partial atomic charges are further screened by the metal). The electrostatic interaction is then dominated by the long-range positive field of the tip, while its negative part close to the oxygen is negligible. For the ionic lattice of $\mathrm{NaCl}$ and the vacancy, the electric field extends much more into the vacuum (for bilayer $\mathrm{NaCl}$ there is also less screening compared to monolayer $\mathrm{hBN}$ ), and thus the interaction with the oxygen's localized negative electric field becomes significant. In addition to these general trends, the effective strengths of the tip dipole and quadrupole moments can vary somewhat in different experiments, which further contributes to the observed contrasts. This suggests that it is important to consider more realistic tip models for some systems and that, in particular, cluster-based models should be used with caution.

To validate the interpretation of boron atoms appearing at larger tip-sample distances than the nitrogens and to provide an additional data channel for elemental identification, we have also performed atomically resolved KPFM experiments. We used a CO-passivated tip to acquire a set of $\Delta f(V)$ spectra $^{65}$ within the area marked with a red square in Figure $6 \mathrm{a}$ and extracted the voltage corresponding to the maximum of the $\Delta f(V)$ parabola, as shown in the example spectra in Figure 6d (see Methods for experimental details). The resulting LCPD map is shown in Figure $6 \mathrm{c}$. In addition, Figure $6 \mathrm{~b}$ displays a simultaneously recorded nc-AFM image that allows comparison with the experiments shown in Figure 2 and the nc-AFM simulations in Figure 5. The LCPD map shows atomic resolution, with a hexagonal pattern of regions of more negative LCPD values. The simultaneously recorded nc-AFM image allows us to match these regions with the sublattice appearing at larger distances in the nc-AFM images, i.e., the boron atoms according to the image simulations. Importantly, Figure $6 \mathrm{~b}$ also shows that the elemental contrast in LCPD is achieved at tip-sample distances that correspond to only weak atomic contrast in nc-AFM images, without the necessity to approach the sample into a regime where the signal is influenced by $\mathrm{CO}$ bending, tip-sample junction instabilities, or $\Delta f(V)$ spectra deviating from their expected parabolic shape. $^{53}$

At these relatively large tip-sample distances, the LCPD contrast is predominantly governed by the vertical component of the electric field of the sample $\left(E_{z}\right){ }^{65}$ In this approximation, a more positive electric field-caused, for example, by partial positive charges-results in a more negative LCPD. Figure $6 \mathrm{e}$ is a map of $E_{z}$ over the entire moiré unit cell, calculated from the Hartree potential of our $\mathrm{hBN} / \operatorname{Ir}(111)$ DFT simulations. The constant-height slice is taken at $3.8 \AA$ above the mean adsorption height of the $\mathrm{hBN}$ layer, i.e., the height of the $\mathrm{CO}$ tip's oxygen atom from the nc-AFM image simulation in Figure $5 c$, which shows good agreement in terms of atomic contrast to Figure $6 \mathrm{~b}$. The reduced work function at the depressions of the moire $^{57}$ results in a long-range modulation of $E_{z}$, with the depressions exhibiting more positive values. Figure $6 f$ is a zoom-in at the region marked by the red square in Figure 6e, corresponding to the region of the experimental LCPD map (see Figure 6a). The theoretical map shows similar elemental contrast to the experimental one, allowing us to identify the regions of more negative LCPD with the boron sublattice. This 

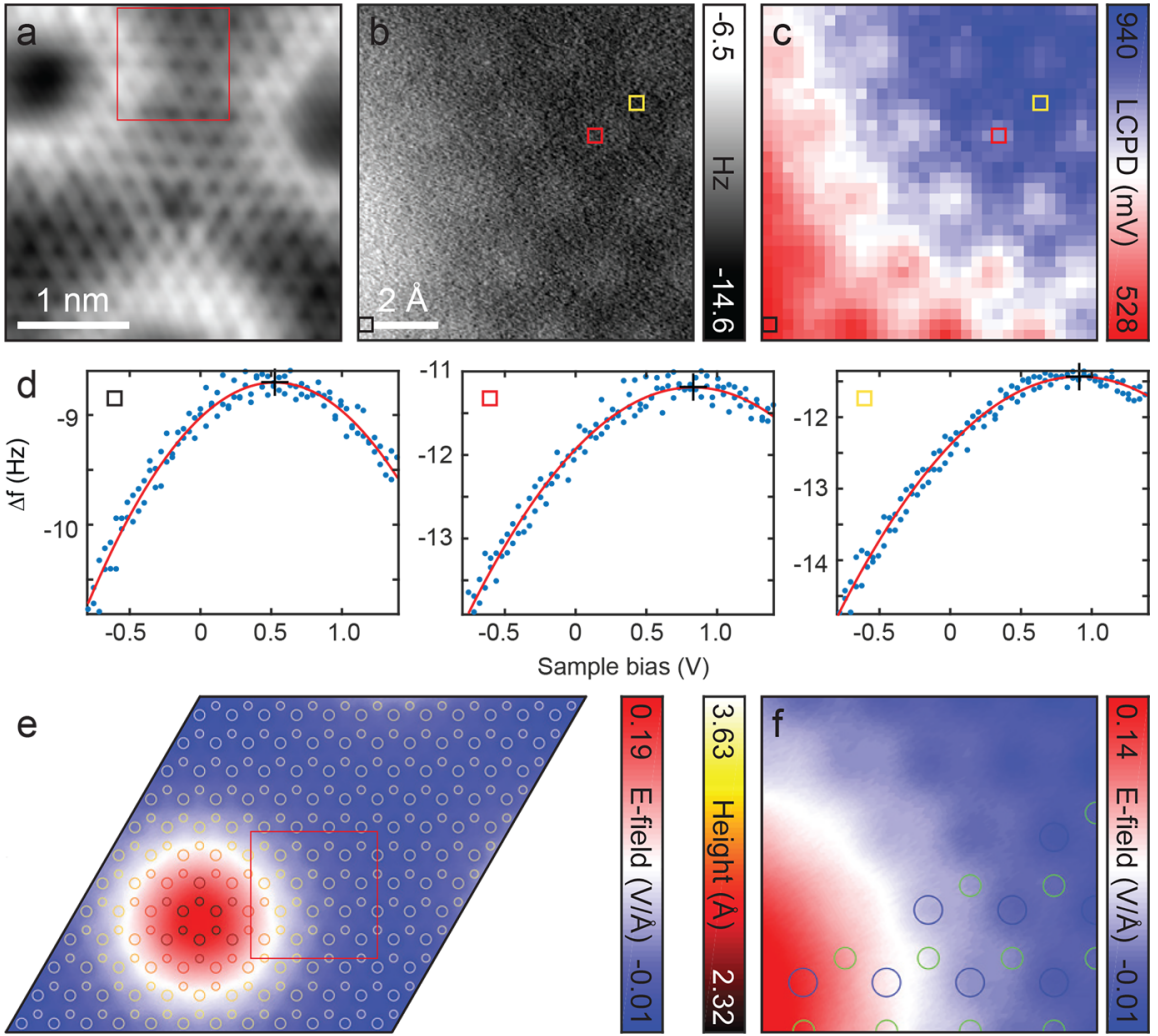

Figure 6. (a) STM image of the hBN moiré. Set point: $0.05 \mathrm{~V}, 0.30 \mathrm{nA}$. (b) Constant-height nc-AFM image of the region marked by the red square in panel (a). Set point: $0.05 \mathrm{~V}, 0.30 \mathrm{nA} ; \Delta z:-0.80 \AA$ A. (c) Simultaneously recorded LCPD map, showing atomic contrast. (d) Three example $\Delta f(V)$ spectra, along with their second-order polynomial fit. Positions in the LCPD map as indicated in panels (b) and (c). (e) Vertical component of the electric field over the moiré unit cell in a plane $3.8 \AA$ above the mean adsorption height of the hBN layer, with overlaid hBN lattice. Large circles: nitrogen, small circles: boron. (f) Zoom-in of the electric field on the region marked with a red square in panel (e), with overlaid hBN lattice. Large blue circles: nitrogen; small green circles: boron.

finding is consistent with the interpretation of the nc-AFM images, thus confirming our assignment for the elements on the two sublattices.

\section{CONCLUSIONS}

We have demonstrated elemental contrast in nc-AFM and KPFM images on monolayer hexagonal boron nitride. Using a passivated tip and a covalently bonded, inert model surface, our results expand the limits of elemental identification with stateof-the-art atomic force microscopy. Most importantly, our method of combining constant-height $\Delta f$ images and KPFM maps presents a robust method to identify different chemical species using nc-AFM.

\section{METHODS}

Experimental Procedures. Monolayers of hBN on $\operatorname{Ir}(111)$ were grown by low-pressure high-temperature chemical vapor deposition of borazine $\left(\mathrm{B}_{3} \mathrm{~N}_{3} \mathrm{H}_{6}\right)$, as described in detail in ref 57 .

All subsequent nc-AFM and STM measurements were carried out in a Createc LT-STM/AFM, operated at ultrahigh vacuum (UHV) and at a temperature of $5 \mathrm{~K}$. The microscope was equipped with a qPlus $^{66}$ tuning fork sensor, which had a resonance frequency $f_{0}$ of $\sim 30.68 \mathrm{kHz}$, a quality factor $Q$ of $\sim 98 k$, and a stiffness $k$ of $\sim 1.8 \mathrm{kN} /$ $\mathrm{m}$. For tip functionalization, $\mathrm{CO}$ was dosed from a leak valve attached to the UHV system onto the cold surface, either $\mathrm{Cu}(111)$ or $\mathrm{hBN} /$ $\operatorname{Ir}(111)$. CO was picked up from $\mathrm{Cu}(111)$ as described previously. ${ }^{67}$ After successful tip passivation, the $\mathrm{Cu}(111)$ sample was exchanged for the $\mathrm{hBN} / \mathrm{Ir}(111)$ sample to carry out the nc-AFM measurements. ${ }^{32}$ On $\operatorname{Ir}(111)$, CO pickup cannot be carried out as well-controlled as on $\mathrm{Cu}(111)$ because of the strong binding of $\mathrm{CO}$ to the Ir surface.
Instead, $\mathrm{CO}$ pickup was achieved by scanning an $\operatorname{Ir}(111)$ surface with high $\mathrm{CO}$ coverage at relatively harsh feedback parameters, e.g., $\sim 10$ $\mathrm{mV}$ sample bias voltage and several tens of $\mathrm{nA}$ tunneling current. This usually led to the transfer of $\mathrm{CO}$ from the surface to the tip apex after some scanning time.

All nc-AFM images and KPFM maps were recorded in the constantheight mode with the $z$-feedback loop disabled and with a tuning fork oscillation amplitude of $50 \mathrm{pm}$. The sample bias voltage for nc-AFM images was $0 \mathrm{~V}$. KPFM measurements were performed by collecting $\Delta f(V)$ spectra on a $32 \times 32$ grid, $^{65}$ where each spectrum took $5 \mathrm{~s}$. The spectra were fitted with second-order polynomials to yield the voltage corresponding to the maxima of the $\Delta f(V)$ parabolas. This voltage minimizes the electrostatic tip-sample forces and is plotted in the LCPD map. Unless stated otherwise, all nc-AFM and KPFM data are unfiltered raw data.

DFT Calculations of $\mathrm{hBN} / \mathrm{Ir}(\mathbf{1 1 1})$. We used the CP2K software package ${ }^{68}$ in particular the QuickStep module, ${ }^{69}$ for the DFT calculations of the structure of $\mathrm{hBN} / \operatorname{Ir}(111)$. The vdW-DF2-B86r approximation $^{70,71}$ from the LibXC library ${ }^{72}$ was employed for the exchange-correlation term in the Kohn-Sham scheme. The Gaussian plane wave method ${ }^{73}$ was used to solve the electronic structure selfconsistently, where the basis set to expand the wave functions was DZVP-MOLOPT-SR-GTH ${ }^{74}$ and a cutoff energy of 700 Ry was used for expanding the density in plane waves, with five grids and a relative cutoff of $70 \mathrm{Ry}$. The Goedecker-Teter-Hutter (GTH) type pseudopotentials $^{75}$ were employed, with 17 valence electrons in Ir. Only the $\Gamma$ point was included in the reciprocal space, without further sampling of the first Brillouin zone. The Fermi-Dirac broadening of the occupation numbers with a "temperature" of $300 \mathrm{~K}$ was used. We used a $12 \times 12$-on- $11 \times 11$ unit cell, in agreement with previous experimental results, ${ }^{57,60}$ and four layers of $\operatorname{Ir}(111)$. Due to the weak 
interactions and strong requirement for precision, the convergency criterion on the maximum force on any ion was set to $0.0514 \mathrm{meV} / \AA$.

DFT Calculations of Different Tip Models. First-principles calculations of the different tip models were performed using the periodic plane-wave basis VASP code ${ }^{76,77}$ implementing the spinpolarized DFT. To accurately include van der Waals interactions in this system, we used the optB86b-vdW-DF functional, ${ }^{78-80}$ selected based on previous work showing that it provides a sufficiently accurate description for all subsystems involved in the measurement. Projected augmented wave potentials were used to describe the core electrons, ${ }^{81}$ with a kinetic energy cutoff of $550 \mathrm{eV}$ (with PREC = accurate). All calculations were performed with dipole correction to account for spurious electrostatic interactions between neighboring cells. Systematic $k$-point convergence was checked for all periodic systems, with sampling chosen according to system size. This approach converged the total energy of all the systems to the order of meV. The properties of the bulk and surface of $\mathrm{Cu}$, Ir, the isolated structure of $\mathrm{CO}$, and its adsorption on $\mathrm{Cu}$ and $\mathrm{Ir}$ were carefully checked within this methodology, and excellent agreement was achieved with experiments. A vacuum gap of at least $1.5 \mathrm{~nm}$ was used in general, with larger gaps to study the tip potentials. All systems considered were relaxed until the atomic forces were less than $0.01 \mathrm{eV} / \AA$.

The tips' electrostatic field was calculated by exploring the electrostatic potential from the DFT simulations. The average electrostatic potential in $x-y$ was calculated as a function of $z$ for each tip model. Beyond the tip apex, this rapidly converges to a constant slope (which reflects the compensation of the electrostatic interactions across the periodic cells and is directly correlated with the tip dipole ${ }^{82}$ ). While the absolute value of this slope is not particularly meaningful, it gives a clear relative measure of the effective electric field at long range. For comparison, the conventionally calculated total dipole in the $z$-direction for each model is shown in the SI, and it shows the same trends across different classes of tip.

nc-AFM Image Simulations. nc-AFM simulations were based on the probe particle model, ${ }^{34,35,41}$ widely used to model the nc-AFM imaging process with functionalized tips, ${ }^{14}$ as implemented in the MechAFM code. ${ }^{62}$ The probe consists of a fixed C atom holder connected to an $\mathrm{O}$ atom restrained in the $x y$-plane by a harmonic spring. The tip-sample interactions were calculated by placing the tip in several locations above the DFT-optimized structure of hBN/ $\operatorname{Ir}(111)$ and relaxing the tip $\mathrm{O}$ termination. The interatomic interactions between $\mathrm{O}$ and the atomic species of the sample are described by Lennard-Jones potentials. For $\mathrm{C}$ and $\mathrm{O}$ atoms, we used the CHARMM force field parameters. ${ }^{83}$ For $\mathrm{B}$ and $\mathrm{N}$ atoms, the parameters from Hilder et al., ${ }^{84}$ derived for the interaction of boron nitride nanotubes with water $\left(\mathrm{H}_{2} \mathrm{O}\right)$, were used. Parameters for pair potentials were obtained using arithmetic mixing rules from the atomic ones. In addition, the MechAFM code allows for inclusion of electrostatic forces by assigning a charge to the $\mathrm{O}$ atom and letting it interact with the Hartree potential of the sample as obtained from DFT. Frequency shift images were calculated from the tip-sample interaction maps using the method in ref 1 , assuming for the $\mathrm{O}$ atom a harmonic spring stiffness of $0.5 \mathrm{~N} / \mathrm{m}$ and a charge of $0.5,0$, or -0.5 . The probe particle model including more complicated tip electrostatics is discussed in the SI.

\section{ASSOCIATED CONTENT}

\section{S Supporting Information}

The Supporting Information is available free of charge on the ACS Publications website at DOI: 10.1021/acsnano.7b08997.

Additional nc-AFM data and simulations and structure of all calculated tips (PDF)

\section{AUTHOR INFORMATION}

\section{Corresponding Author}

*E-mail: peter.liljeroth@aalto.fi.

\section{ORCID}

Fabian Schulz: 0000-0002-1359-4675

Ari Paavo Seitsonen: 0000-0003-4331-0650

Adam S. Foster: 0000-0001-5371-5905

Peter Liljeroth: 0000-0003-1253-8097

\section{Present Address}

\#IBM Research Laboratory Zurich, Säumerstrasse 4, CH-8803 Rüschlikon, Switzerland.

\section{Notes}

The authors declare no competing financial interest.

\section{ACKNOWLEDGMENTS}

We thank Leo Gross and Nikolaj Moll for valuable scientific discussions and John Tracey for support with the nc-AFM simulations. O.K. appreciates help from Prokop Hapala on the programming of the enhanced probe particle code. This research made use of the Aalto Nanomicroscopy Center (Aalto NMC) facilities and was supported by the European Research Council (ERC-2011-StG No. 278698 "PRECISENANO") and the Academy of Finland (Project nos. 305635, 311012, 314877, and 314882, and Centres of Excellence Program project nos. 284594 and 284621). J.R. and A.S.F. were supported by EU project PAMS (contract no. 610446), and acknowledge use of the CSC, Helsinki, for computational resources. A.P.S. acknowledges the computational resources at CSC, Espoo, project 2000606, and Centro Svizzero di Calcolo Scientifico (CSCS), Lugano, project uzh11.

\section{REFERENCES}

(1) Giessibl, F. J. Advances in Atomic Force Microscopy. Rev. Mod. Phys. 2003, 75, 949-983.

(2) Chen, C. J. Introduction to Scanning Tunneling Microscopy, 2nd ed.; Oxford University Press: Oxford, 2008.

(3) Morita, S., Giessibl, E., Meyer, F. J., Wiesendanger, R., Eds. Noncontact Atomic Force Microscopy; Springer: Berlin, 2015; Vol. 3.

(4) Oxley, M. P.; Lupini, A. R.; Pennycook, S. J. Ultra-High Resolution Electron Microscopy. Rep. Prog. Phys. 2017, 80, 026101.

(5) Foster, A. S.; Barth, C.; Shluger, A. L.; Reichling, M. Unambiguous Interpretation of Atomically Resolved Force Microscopy Images of an Insulator. Phys. Rev. Lett. 2001, 86, 2373-2376.

(6) Hofer, W. A.; Foster, A. S.; Shluger, A. L. Theories of Scanning Probe Microscopes at the Atomic Scale. Rev. Mod. Phys. 2003, 75, 1287-1331.

(7) Sugimoto, Y.; Pou, P.; Abe, M.; Jelínek, P.; Pérez, R.; Morita, S.; Custance, O. Chemical Identification of Individual Surface Atoms by Atomic Force Microscopy. Nature 2007, 446, 64-67.

(8) Kimoto, K.; Asaka, T.; Nagai, T.; Saito, M.; Matsui, Y.; Ishizuka, $\mathrm{K}$. Element-Selective Imaging of Atomic Columns in a Crystal Using STEM and EELS. Nature 2007, 450, 702-704.

(9) Muller, D. A.; Kourkoutis, L. F.; Murfitt, M.; Song, J. H.; Hwang, H. Y.; Silcox, J.; Dellby, N.; Krivanek, O. L. Atomic-Scale Chemical Imaging of Composition and Bonding by Aberration-Corrected Microscopy. Science 2008, 319, 1073-1076.

(10) Zhou, W.; Kapetanakis, M. D.; Prange, M. P.; Pantelides, S. T.; Pennycook, S. J.; Idrobo, J.-C. Direct Determination of the Chemical Bonding of Individual Impurities in Graphene. Phys. Rev. Lett. 2012, 109, 206803.

(11) Ramasse, Q. M.; Seabourne, C. R.; Kepaptsoglou, D.-M.; Zan, R.; Bangert, U.; Scott, A. J. Probing the Bonding and Electronic Structure of Single Atom Dopants in Graphene with Electron Energy Loss Spectroscopy. Nano Lett. 2013, 13, 4989-4995.

(12) Krivanek, O. L.; Chisholm, M. F.; Nicolosi, V.; Pennycook, T. J.; Corbin, G. J.; Dellby, N.; Murfitt, M. F.; Own, C. S.; Szilagyi, Z. S.; Oxley, M. P.; Pantelides, S. T.; Pennycook, S. J. Atom-by-Atom 
Structural and Chemical Analysis by Annular Dark-Field Electron Microscopy. Nature 2010, 464, 571-574.

(13) Pavliček, N.; Gross, L. Generation, Manipulation and Characterization of Molecules by Atomic Force Microscopy. Nat. Rev. Chem. 2017, 1, 0005.

(14) Jelínek, P. High Resolution SPM Imaging of Organic Molecules with Functionalized Tips. J. Phys.: Condens. Matter 2017, 29, 343002.

(15) Albrecht, T. R.; Grütter, P.; Horne, D.; Rugar, D. FrequencyModulation Detection Using High-Q Cantilevers for Enhanced Force Microscope Sensitivity. J. Appl. Phys. 1991, 69, 668-673.

(16) Barth, C.; Foster, A. S.; Henry, C. R.; Shluger, A. L. Recent Trends in Surface Characterization and Chemistry with HighResolution Scanning Force Methods. Adv. Mater. 2011, 23, 477-501.

(17) Stróżecka, A.; Li, J.; Schürmann, R.; Schulze, G.; Corso, M.; Schulz, F.; Lotze, C.; Sadewasser, S.; Franke, K. J.; Pascual, J. I. Electroluminescence of Copper-Nitride Nanocrystals. Phys. Rev. B: Condens. Matter Mater. Phys. 2014, 90, 195420.

(18) Schneiderbauer, M.; Emmrich, M.; Weymouth, A. J.; Giessibl, F. J. CO Tip Functionalization Inverts Atomic Force Microscopy Contrast via Short-Range Electrostatic Forces. Phys. Rev. Lett. 2014, 112,166102 .

(19) Heyde, M.; Sterrer, M.; Rust, H. P.; Freund, H.-J. Atomic Resolution on $\mathrm{MgO}(001)$ by Atomic Force Microscopy Using a Double Quartz Tuning Fork Sensor at Low-Temperature and Ultrahigh Vacuum. Appl. Phys. Lett. 2005, 87, 083104.

(20) Onoda, J.; Ondráček, M.; Jelínek, P.; Sugimoto, Y. Electronegativity Determination of Individual Surface Atoms by Atomic Force Microscopy. Nat. Commun. 2017, 8, 15155.

(21) Bocquet, F.; Nony, L.; Loppacher, C.; Glatzel, T. Analytical Approach to the Local Contact Potential Difference on (001) Ionic Surfaces: Implications for Kelvin Probe Force Microscopy. Phys. Rev. B: Condens. Matter Mater. Phys. 2008, 78, 035410.

(22) Enevoldsen, G. H.; Glatzel, T.; Christensen, M. C.; Lauritsen, J. V.; Besenbacher, F. Atomic Scale Kelvin Probe Force Microscopy Studies of the Surface Potential Variations on the $\mathrm{TiO}_{2}(110)$ Surface. Phys. Rev. Lett. 2008, 100, 236104.

(23) Yurtsever, A.; Sugimoto, Y.; Fukumoto, M.; Abe, M.; Morita, S. Effect of Tip Polarity on Kelvin Probe Force Microscopy Images of Thin Insulator $\mathrm{CaF}_{2}$ Films on $\mathrm{Si}(111)$. Appl. Phys. Lett. 2012, 101, 083119.

(24) Gross, L.; Schuler, B.; Mohn, F.; Moll, N.; Pavliček, N.; Steurer, W.; Scivetti, I.; Kotsis, K.; Persson, M.; Meyer, G. Investigating Atomic Contrast in Atomic Force Microscopy and Kelvin Probe Force Microscopy on Ionic Systems Using Functionalized Tips. Phys. Rev. B: Condens. Matter Mater. Phys. 2014, 90, 155455.

(25) Sugawara, Y.; Uchihashi, T.; Abe, M.; Morita, S. True Atomic Resolution Imaging of Surface Structure and Surface Charge on the GaAs(110). Appl. Surf. Sci. 1999, 140, 371-375.

(26) Sadewasser, S.; Jelínek, P.; Fang, C.-K.; Custance, O.; Yamada, Y.; Sugimoto, Y.; Abe, M.; Morita, S. New Insights on AtomicResolution Frequency-Modulation Kelvin-Probe Force-Microscopy Imaging of Semiconductors. Phys. Rev. Lett. 2009, 103, 266103.

(27) Okamoto, K.; Yoshimoto, K.; Sugawara, Y.; Morita, S. KPFM Imaging of $\mathrm{Si}(111) 5 \sqrt{3} \times 5 \sqrt{3}$-Sb Surface for Atom Distinction Using NC-AFM. Appl. Surf. Sci. 2003, 210, 128-133.

(28) Gross, L.; Mohn, F.; Moll, N.; Liljeroth, P.; Meyer, G. The Chemical Structure of a Molecule Resolved by Atomic Force Microscopy. Science 2009, 325, 1110-1114.

(29) Gross, L.; Mohn, F.; Moll, N.; Meyer, G.; Ebel, R.; AbdelMageed, W. M.; Jaspars, M. Organic Structure Determination Using Atomic-Resolution Scanning Probe Microscopy. Nat. Chem. 2010, 2, $821-825$.

(30) Sun, Z.; Boneschanscher, M. P.; Swart, I.; Vanmaekelbergh, D.; Liljeroth, P. Quantitative Atomic Force Microscopy with Carbon Monoxide Terminated Tips. Phys. Rev. Lett. 2011, 106, 046104.

(31) Gross, L.; Mohn, F.; Moll, N.; Schuler, B.; Criado, A.; Guitian, E.; Pena, D.; Gourdon, A.; Meyer, G. Bond-Order Discrimination by Atomic Force Microscopy. Science 2012, 337, 1326-1329.
(32) Boneschanscher, M. P.; van der Lit, J.; Sun, Z.; Swart, I.; Liljeroth, P.; Vanmaekelbergh, D. Quantitative Atomic Resolution Force Imaging on Epitaxial Graphene with Reactive and Non-Reactive AFM Probes. ACS Nano 2012, 6, 10216-10221.

(33) Mönig, H.; Hermoso, D. R.; Díaz Arado, O.; Todorović, M.; Timmer, A.; Schüer, S.; Langewisch, G.; Pérez, R.; Fuchs, H. Submolecular Imaging by Noncontact Atomic Force Microscopy with an Oxygen Atom Rigidly Connected to a Metallic Probe. ACS Nano 2016, 10, 1201-1209.

(34) Hämäläinen, S. K.; van der Heijden, N.; van der Lit, J.; den Hartog, S.; Liljeroth, P.; Swart, I. Intermolecular Contrast in Atomic Force Microscopy Images without Intermolecular Bonds. Phys. Rev. Lett. 2014, 113, 186102 .

(35) Hapala, P.; Kichin, G.; Wagner, C.; Tautz, F. S.; Temirov, R.; Jelínek, P. Mechanism of High-Resolution STM/AFM Imaging with Functionalized Tips. Phys. Rev. B: Condens. Matter Mater. Phys. 2014, 90, 085421.

(36) Schulz, F.; Jacobse, P. H.; Canova, F. F.; van der Lit, J.; Gao, D. Z.; van den Hoogenband, A.; Han, P.; Klein Gebbink, R. J. M.; Moret, M.-E.; Joensuu, P. M.; Swart, I.; Liljeroth, P. Precursor Geometry Determines the Growth Mechanism in Graphene Nanoribbons. J. Phys. Chem. C 2017, 121, 2896-2904.

(37) Moll, N.; Gross, L.; Mohn, F.; Curioni, A.; Meyer, G. A Simple Model of Molecular Imaging with Noncontact Atomic Force Microscopy. New J. Phys. 2012, 14, 083023.

(38) Guo, C.-S.; Van Hove, M. A.; Ren, X.; Zhao, Y. High-Resolution Model for Noncontact Atomic Force Microscopy with a Flexible Molecule on the Tip Apex. J. Phys. Chem. C 2015, 119, 1483-1488.

(39) Sakai, Y.; Lee, A. J.; Chelikowsky, J. R. First-Principles Atomic Force Microscopy Image Simulations with Density Embedding Theory. Nano Lett. 2016, 16, 3242-3246.

(40) Hämäläinen, S. K.; Boneschanscher, M. P.; Jacobse, P. H.; Swart, I.; Pussi, K.; Moritz, W.; Lahtinen, J.; Liljeroth, P.; Sainio, J. Structure and Local Variations of the Graphene Moire on $\operatorname{Ir}(111)$. Phys. Rev. B: Condens. Matter Mater. Phys. 2013, 88, 201406.

(41) Boneschanscher, M. P.; Hämäläinen, S. K.; Liljeroth, P.; Swart, I. Sample Corrugation Affects the Apparent Bond Lengths in Atomic Force Microscopy. ACS Nano 2014, 8, 3006-3014.

(42) Koch, S.; Langer, M.; Kawai, S.; Meyer, E.; Glatzel, T. Contrast Inversion of the h-BN Nanomesh Investigated by NC-AFM and Kelvin Probe Force Microscopy. J. Phys.: Condens. Matter 2012, 24, 314212.

(43) Herden, T.; Ternes, M.; Kern, K. Lateral and Vertical Stiffness of the Epitaxial h-BN Monolayer on $\mathrm{Rh}(111)$. Nano Lett. 2014, 14, 3623-3627.

(44) Schwarz, M.; Riss, A.; Garnica, M.; Ducke, J.; Deimel, P. S.; Duncan, D. A.; Thakur, P. K.; Lee, T.-L.; Seitsonen, A. P.; Barth, J. V.; Allegretti, F.; Auwärter, W. Corrugation in the Weakly Interacting Hexagonal-BN/Cu(111) System: Structure Determination by Combining Noncontact Atomic Force Microscopy and X-Ray Standing Waves. ACS Nano 2017, 11, 9151-9161.

(45) Schwarz, A.; Koehler, A.; Grenz, J.; Wiesendanger, R. Detecting the Dipole Moment of a Single Carbon Monoxide Molecule. Appl. Phys. Lett. 2014, 105, 011606.

(46) van der Lit, J.; Di Cicco, F.; Hapala, P.; Jelínek, P.; Swart, I. Submolecular Resolution Imaging of Molecules by Atomic Force Microscopy: The Influence of the Electrostatic Force. Phys. Rev. Lett. 2016, 116, 096102.

(47) Hapala, P.; Švec, M.; Stetsovych, O.; van der Heijden, N. J.; Ondráček, M.; van der Lit, J.; Mutombo, P.; Swart, I.; Jelínek, P. Mapping the Electrostatic Force Field of Single Molecules from HighResolution Scanning Probe Images. Nat. Commun. 2016, 7, 11560.

(48) Ellner, M.; Pavliček, N.; Pou, P.; Schuler, B.; Moll, N.; Meyer, G.; Gross, L.; Pérez, R. The Electric Field of CO Tips and Its Relevance for Atomic Force Microscopy. Nano Lett. 2016, 16, 19741980.

(49) Hanssen, K. O.; Schuler, B.; Williams, A. J.; Demissie, T. B.; Hansen, E.; Andersen, J. H.; Svenson, J.; Blinov, K.; Repisky, M.; Mohn, F.; Meyer, G.; Svendsen, J.-S.; Ruud, K.; Elyashberg, M.; Gross, 
L.; Jaspars, M.; Isaksson, J. A Combined Atomic Force Microscopy and Computational Approach for the Structural Elucidation of Breitfussin A and B: Highly Modified Halogenated Dipeptides from Thuiaria Breitfussi. Angew. Chem., Int. Ed. 2012, 51, 12238-12241.

(50) Schuler, B.; Liu, S.-X.; Geng, Y.; Decurtins, S.; Meyer, G.; Gross, L. Contrast Formation in Kelvin Probe Force Microscopy of Single $\pi$ Conjugated Molecules. Nano Lett. 2014, 14, 3342-3346.

(51) van der Heijden, N. J.; Hapala, P.; Rombouts, J. A.; van der Lit, J.; Smith, D.; Mutombo, P.; Švec, M.; Jelínek, P.; Swart, I. Characteristic Contrast in $\Delta f_{\min }$ Maps of Organic Molecules Using Atomic Force Microscopy. ACS Nano 2016, 10, 8517-8525.

(52) Kawai, S.; Saito, S.; Osumi, S.; Yamaguchi, S.; Foster, A. S.; Spijker, P.; Meyer, E. Atomically Controlled Substitutional BoronDoping of Graphene Nanoribbons. Nat. Commun. 2015, 6, 8098.

(53) Albrecht, F.; Repp, J.; Fleischmann, M.; Scheer, M.; Ondráček, M.; Jelínek, P. Probing Charges on the Atomic Scale by Means of Atomic Force Microscopy. Phys. Rev. Lett. 2015, 115, 076101.

(54) Neu, M.; Moll, N.; Gross, L.; Meyer, G.; Giessibl, F. J.; Repp, J. Image Correction for Atomic Force Microscopy Images with Functionalized Tips. Phys. Rev. B: Condens. Matter Mater. Phys. 2014, 89, 205407.

(55) Moll, N.; Schuler, B.; Kawai, S.; Xu, F.; Peng, L.; Orita, A.; Otera, J.; Curioni, A.; Neu, M.; Repp, J.; Meyer, G.; Gross, L. Image Distortions of a Partially Fluorinated Hydrocarbon Molecule in Atomic Force Microscopy with Carbon Monoxide Terminated Tips. Nano Lett. 2014, 14, 6127-6131.

(56) Kawai, S.; Nishiuchi, T.; Kodama, T.; Spijker, P.; Pawlak, R.; Meier, T.; Tracey, J.; Kubo, T.; Meyer, E.; Foster, A. S. Direct Quantitative Measurement of the $\mathrm{C}=\mathrm{O} \cdots \mathrm{H}-\mathrm{C}$ Bond by Atomic Force Microscopy. Sci. Adv. 2017, 3, e1603258.

(57) Schulz, F.; Drost, R.; Hämäläinen, S. K.; Demonchaux, T.; Seitsonen, A. P.; Liljeroth, P. Epitaxial Hexagonal Boron Nitride on Ir(111): A Work Function Template. Phys. Rev. B: Condens. Matter Mater. Phys. 2014, 89, 235429.

(58) Karch, K.; Bechstedt, F. Ab Initio Lattice Dynamics of BN and AlN: Covalent Versus Ionic Forces. Phys. Rev. B: Condens. Matter Mater. Phys. 1997, 56, 7404-7415.

(59) Ooi, N.; Rairkar, A.; Lindsley, L.; Adams, J. B. Electronic Structure and Bonding in Hexagonal Boron Nitride. J. Phys.: Condens. Matter 2006, 18, 97-115.

(60) zum Hagen, F. H. F.; Zimmermann, D. M.; Silva, C. C.; Schlueter, C.; Atodiresei, N.; Jolie, W.; Martínez-Galera, A. J.; Dombrowski, D.; Schröder, U. A.; Will, M.; Lazić, P.; Caciuc, V.; Blügel, S.; Lee, T.-L.; Michely, T.; Busse, C. Structure and Growth of Hexagonal Boron Nitride on $\operatorname{Ir}(111)$. ACS Nano 2016, 10, 11012110261.

(61) Sader, J. E.; Jarvis, S. P. Accurate Formulas for Interaction Force and Energy in Frequency Modulation Force Spectroscopy. Appl. Phys. Lett. 2004, 84, 1801-1803.

(62) Tracey, J.; Canova, F. F.; Keisanen, O.; Gao, D. Z.; Spijker, P.; Reischl, B.; Foster, A. S. Flexible and Modular Virtual Scanning Probe Microscope. Comput. Phys. Commun. 2015, 196, 429-438.

(63) Slater, J. Atomic Radii in Crystals. J. Chem. Phys. 1964, 41, 3199-3204.

(64) Gao, D. Z.; Grenz, J.; Watkins, M. B.; Federici Canova, F.; Schwarz, A.; Wiesendanger, R.; Shluger, A. L. Using Metallic Noncontact Atomic Force Microscope Tips for Imaging Insulators and Polar Molecules: Tip Characterization and Imaging Mechanisms. ACS Nano 2014, 8, 5339-5351.

(65) Mohn, F.; Gross, L.; Moll, N.; Meyer, G. Imaging the Charge Distribution Within a Single Molecule. Nat. Nanotechnol. 2012, 7, 227-231.

(66) Giessibl, F. J. High-Speed Force Sensor for Force Microscopy and Profilometry Utilizing a Quartz Tuning Fork. Appl. Phys. Lett. 1998, 73, 3956-3958.

(67) Bartels, L.; Meyer, G.; Rieder, K.-H. Controlled Vertical Manipulation of Single CO Molecules with the Scanning Tunneling Microscope: A Route to Chemical Contrast. Appl. Phys. Lett. 1997, 71, 213-215.
(68) The CP2K Developers' Group; CP2K is freely available from http://www.cp2k.org/ (accessed October 2017).

(69) VandeVondele, J.; Krack, M.; Mohamed, F.; Parrinello, M.; Chassaing, T.; Hutter, J. QUICKSTEP: Fast and Accurate Density Functional Calculations Using a Mixed Gaussian and Plane Waves Approach. Comput. Phys. Commun. 2005, 167, 103-128.

(70) Hamada, I. van der Waals Density Functional Made Accurate. Phys. Rev. B: Condens. Matter Mater. Phys. 2014, 89, 121103.

(71) Lee, K.; Murray, D. E.; Kong, L.; Lundqvist, B. I.; Langreth, D. C. Higher-Accuracy van der Waals Density Functional. Phys. Rev. B: Condens. Matter Mater. Phys. 2010, 82, 081101.

(72) Marques, M. A. L.; Oliveira, M. J. T.; Burnus, T. Libxc: A Library of Exchange and Correlation Functionals for Density Functional Theory. Comput. Phys. Commun. 2012, 183, 2272-2281.

(73) Lippert, G.; Hutter, J.; Parrinello, M. A Hybrid Gaussian and Plane Wave Density Functional Scheme. Mol. Phys. 1997, 92, 477487.

(74) VandeVondele, J.; Hutter, J. Gaussian Basis Sets for Accurate Calculations on Molecular Systems in Gas and Condensed Phases. J. Chem. Phys. 2007, 127, 114105.

(75) Goedecker, S.; T, M.; Hutter, J. Separable Dual-Space Gaussian Pseudopotentials. Phys. Rev. B: Condens. Matter Mater. Phys. 1996, 54, $1703-1710$

(76) Kresse, G.; Furthmüller, J. Efficiency of Ab-Initio Total Energy Calculations for Metals and Semiconductors Using a Plane-Wave Basis Set. Comput. Mater. Sci. 1996, 6, 15-50.

(77) Kresse, G.; Furthmüller, J. Efficient Iterative Schemes for $A b$ Initio Total-Energy Calculations Using a Plane-Wave Basis Set. Phys. Rev. B: Condens. Matter Mater. Phys. 1996, 54, 11169-11186.

(78) Klimeš, J.; Bowler, D. R.; Michaelides, A. Chemical Accuracy for the van der Waals Density Functional. J. Phys.: Condens. Matter 2010, 22, 022201.

(79) Klimeš, J.; Bowler, D. R.; Michaelides, A. Van der Waals Density Functionals Applied to Solids. Phys. Rev. B: Condens. Matter Mater. Phys. 2011, 83, 195131.

(80) Björkman, T.; Gulans, A.; Krasheninnikov, A.; Nieminen, R. van der Waals Bonding in Layered Compounds from Advanced DensityFunctional First-Principles Calculations. Phys. Rev. Lett. 2012, 108, 235502.

(81) Blöchl, P. E. Projector Augmented-Wave Method. Phys. Rev. B: Condens. Matter Mater. Phys. 1994, 50, 17953-17979.

(82) Natan, A.; Kronik, L.; Shapira, Y. Computing Surface Dipoles and Potentials of Self-Assembled Monolayers from First Principles. Appl. Surf. Sci. 2006, 252, 7608-7613.

(83) Vanommeslaeghe, K.; MacKerell, A. D. Automation of the CHARMM General Force Field (CGenFF) I: Bond Perception and Atom Typing. J. Chem. Inf. Model. 2012, 52, 3144-3154.

(84) Hilder, T. A.; Yang, R.; Ganesh, V.; Gordon, D.; Bliznyuk, A.; Rendell, A. P.; Chung, S. H. Validity of Current Force Fields for Simulations on Boron Nitride Nanotubes. Micro Nano Lett. 2010, 5, $150-156$. 